\title{
EFEITOS DA INGESTÃO ALCOÓLICA NA FREQUÊNCIA CARDÍACA, POTÊNCIA E TEMPO NO ESFORÇO MÁXIMO
}

\section{Giuliano Roberto da Silva}

Doutorando Strictu Sensu em Promoção de Saúde na Universidade de Franca (UNIFRAN), SP; Docente nas instituições: Faculdade Presbiteriana Gammon (FAGAMMON) - MG, Universidade José do Rosário Vellano (UNIFENAS) - MG, Universidade Vale do Rio Verde (UNINCOR) - MG, Centro Universitário do Sul de Minas Gerais (UNISMG) - MG e Rede Estadual de Ensino (MINAS GERAIS) - MG -

E-mail: giumusc@gmail.com

\section{Gerusa Dias Siqueira Vilela Terra}

Doutoranda em Promoção à Saúde; Coordenadora dos cursos de Educação Física e Nutrição da Universidade José do Rosário Vellano (UNIFENAS), Alfenas (MG), Brasil.

\section{Marcelo Rodrigo Tavares}

Doutor em Ciências Médicas pela Universidade de São Paulo. Professor da Universidade José do Rosário Vellano (UNIFENAS), Alfenas (MG), Brasil

\section{Cassiano Merussi Neiva}

Doutorado em Biologia Funcional e Molecular (Fisiologia e Bioquímica) pela Universidade Estadual de Campinas (UNICAMP) com estágio sanduíche (bolsista do Programa de Doutorado com Estágio no Exterior PDEE) pela Second Medical Faculty - Charles University de Praga, República Checa; Livre Docente; Docente e Tutor de Graduação em Medicina, Nutrição, Educação Física, Fisioterapia, Biologia e Psicologia.

\section{César Augusto Costa Rodrigues}

Mestrado em Saúde Coletiva; Docente da Universidade José do Rosário Vellano (UNIFENAS), Alfenas (MG), Brasil.

\section{Yvan Fernandes Vilas Boas}

Docente efetivo das Universidade Vale do Rio Verde e Universidade José do Rosário Vellano (UNIFENAS), Alfenas (MG), Brasil; Diretor Escolar na cidade de Caxambu - MG.

\section{Lucas Jacintho de Mendonça Uchôa}

Graduado em Educação Física (Bacharelado) pela Universidade José do Rosário Vellano (UNIFENAS), Alfenas (MG), Brasil.

Os autores declaram inexistência de conflitos de interesses na realização deste trabalho.
RESUMO: O consumo de bebidas alcoólicas entre desportistas é frequente e sabe-se que o álcool é usado como fonte de energia, porém em atividades físicas intensas, que exigem uma grande disponibilidade calórica, o uso pode ser prejudicial para o rendimento. Este estudo teve como objetivo verificar os efeitos da ingestão alcoólica na frequência cardíaca, potência e tempo no esforço máximo de indivíduos jovens não sedentários. Uma amostra de 10 voluntários foi selecionada e foram submetidos ao teste ergométrico inicialmente sem ingestão de álcool. Após o primeiro teste os sujeitos foram instruídos a ficarem sete dias sem ingerir álcool, assim, no dia do segundo teste ergométrico, eles ingeriram $0,5 \mathrm{ml}$ de etanol por quilograma de massa magra, 30 minutos antes do teste. Os resultados demonstraram uma diferença estatística $p=0,0058$ no tempo de teste de esforço máximo e $p=$ 0,015 na potência máxima atingida, contudo não houve diferenças estatisticamente significativas $(\mathrm{p}=0,1)$ ao avaliar a frequência cardíaca nos dois testes. Logo, o estudo demonstrou que a ingestão alcoólica interferiu negativamente no desempenho físico.

PALAVRAS-CHAVE: Álcool; Cicloergômetro; Desempenho Físico; Exercício de Exaustão.

\section{EFFECTS OF ALCOHOLIC BEVERAGES ON HEART FREQUENCY, POTENCY AND MAXIMUM EFFORT TIME}

ABSTRACT: The intake of alcoholic beverages among sportsmen is frequent. Although alcohol is an important energy source, its use may bring liabilities in performance in intense physical activities which require great caloric availability. Current study analyzes the effects of alcohol intake on heart frequency, potency and maximum effort time in exercising young people. Ten volunteers were selected and they underwent the ergometric test without any alcohol intake. After the first test, the volunteers were asked to remain seven days without ingesting alcohol beverages. On the second ergometric test they took $0.5 \mathrm{~mL}$ ethanol per $\mathrm{kg}$ of mass, 30 min prior to test. Results revealed a statistical difference, $\mathrm{p}=0.0058$, in maximum effort test and $p=0.015$ in maximum potency, with no significantly statistical difference $(p=0.1)$ when heart frequency was evaluated for the two tests. Analysis demonstrated that alcohol intake interfered negatively on physical performance.

KEYWORDS: Alcohol; Cycle-ergometer; Physical Performance; Exhaustion Exercise. 


\section{INTRODUÇÃO}

Atividade física é entendida como qualquer movimento corporal realizado pela musculatura esquelética, que leve a um gasto energético acima do repouso (CASPERSSEN; POWELL; CHRSITENSON, 1985; NAHAS, 2001). Exige uma grande disponibilidade calórica de um praticante, principalmente em esportes profissionais, e o treinamento de alta intensidade exige o máximo de um atleta (PALACIOS, 2000). É reconhecida por seus efeitos saudáveis em seus praticantes, de forma regular; também por ser associada com diversos benefícios para a saúde é considerada um importante componente de um estilo de vida saudável (LAMONTE; AINSWORTH, 2001; PATE et al., 1995; WORLD HEALTH ORGANIZATION, 2005).

Ter uma boa alimentação e seguir corretamente a dieta de treino é essencial. A American Dietetic Association, Dietitians of Canada e American College of Sports Medicine (ACSM) dizem que a melhora da performance e a recuperação dos desgastes do exercício físico são reforçados por uma alimentação ideal, e recomendam a seleção de alimentos e fluidos saudáveis, horário da alimentação e em alguns casos suplementos para otimizar a saúde e desempenho físico. Os princípios de uma boa dieta e nutrição adequada são os mesmos para pessoas que não praticam esportes e para não atletas (RODRIGUEZ; DIMARCO; LANGLEY, 2009).

A principal diferença está na maior quantidade de energia que desportistas precisam para praticar atividades físicas mais intensas, manter o peso apropriado para permitir melhor performance e manter bom desempenho físico, sem riscos à saúde (PALACIOS, 2000). Porém, o consumo de bebidas alcoólicas vem aumentando no Brasil, segundo o relatório da $\mathrm{ONU}$, inclusive entre desportistas (ORGANIZAÇÂO MUNDIAL DA SAÚDE, 2001). O consumo de álcool está muitas vezes associado intimamente ao esporte (SHIRREFFS; MAUGHAN, 2006). Um estudo apontou três principais motivos pelos quais atletas faziam uso de bebidas alcóolicas, e os classificou em reforço positivo, time/grupo e estresse relacionado ao esporte (MARTENS et al., 2005).

O álcool é a única droga que pode ser classificada como alimento, por ser uma fonte rica em calorias e fornecer grande quantidade de energia para o corpo, é usada pelas células no desempenho de suas funções complicadas; contudo, ao contrário da maioria dos alimentos, o álcool contém quantidades desprezíveis de vitaminas e minerais e contribui pouco ou nada para os requisitos de nutrição das células. Entretanto, é uma fonte de energia diferente das outras, pois não pode ser estocado no organismo (MILAM; KETCHAM, 1991). Como uma substância tóxica, deve ser eliminado imediatamente. Assim, o álcool tem prioridades no metabolismo, alterando outras vias metabólicas, incluindo a oxidação lipídica, o que favorece o estoque de gorduras no organismo, que se depositam em sua maioria na área abdominal (SUTTER, 2005). O álcool em pequenas quantidades é um estimulante regozijador, porém em grandes quantidades é um agente venoso e tóxico (MILAM; KETCHAM, 1991). Desta forma, o objetivo do estudo foi avaliar os efeitos de uma ingestão aguda de álcool na pré-sessão de esforço progressivo em cicloergômetro estacionário sobre as variáveis potência máxima, tempo de permanência no teste e comportamento da frequência cardíaca.

\section{MATERIAL E MÉTODOS}

Trata-se de um estudo longitudinal prospectivo, descritivo do tipo experimental. A pesquisa foi realizada no laboratório de práticas esportivas da Universidade José do Rosário Vellano (UNIFENAS), e aprovada pelo Comitê de Ética em Pesquisa da mesma instituição, com o Parecer de $n^{0}$ 376.398. Foram selecionados para participar voluntariamente da pesquisa 10 indivíduos hígidos, sendo 06 homens e 04 mulheres com idade média de 22,7 $\pm 2,8$ anos, que foram orientados sobre o Termo de Consentimento Livre e Esclarecido (TCLE), bem como sobre os riscos e benefícios da pesquisa, sendo considerados aceitos ao estudo aqueles que, de forma livre, assinaram o TCLE. Todos os procedimentos que foram adotados nesta pesquisa obedeceram aos critérios da Ética em Pesquisa com Seres Humanos conforme Resolução no 466, de dezembro de 2012, do Conselho Nacional de Saúde (CNS).

Todos os voluntários foram submetidos a uma anamnese sobre comportamento físico, alimentar e existência prévia de patologia diagnosticada. 
Além disso, foram avaliados para adipometria, pelo protocolo de Jackson e Pollock (JACKSON; POLLOCK, 1978; JACKSON; POLLOCK; WARD, 1980), utilizando adipômetro clínico SANNY®. Foram também submetidos ao teste cicloergômetro Maxx ${ }^{\circledR}$ (Hidrofit, Brasil), padrão Monark $\AA$, sempre no mesmo horário do dia ( \pm 2 horas) após a última refeição intermediária.

Tiveram a frequência cardíaca monitorada através do frequencímetro Pollar FT1®. Todos os participantes foram instruídos a chegarem ao laboratório em um estado descansado, hidratados, e ainda sem terem realizado nenhum tipo de exercícios exaustivo de membro inferior em até 72 horas antes do teste, além de manter um padrão de sono semelhante, durante as 48 horas antes do teste.

Os voluntários fizeram alongamento de membro inferior durante 30 segundos e aquecimento durante 7 minutos no cicloergômetro mantendo uma cadência entre 40rpm e 60rpm antes do teste se iniciar. Durante o teste era acrescida carga $(50 \mathrm{~W})$ a cada 2 minutos. Foi necessário o participante manter uma cadência entre 70rpm e 90rpm até exaustão ou até não conseguir mais se manter nesta faixa de cadência, sem ingestão de etanol. A frequência cardíaca era verificada a cada minuto.

Após o primeiro teste, todos os participantes foram instruídos a ficarem sete dias sem ingerir bebida alcóolica para serem avaliados no segundo teste; assim, no dia do segundo teste cicloergômetro, eles ingeriram uma quantidade de cerveja contendo o equivalente a 0,5 $\mathrm{ml}$ de etanol por quilograma de massa magra, 30 minutos antes do teste, pois a maior concentração no sangue ocorre entre 30 minutos a 1 hora e 30 minutos após a ingestão (OGA, 1996). Os dados obtidos de frequência cardíaca, potência e tempo máximo de exaustão ao exercício foram submetidos a uma análise estatística com o teste T pareado, com nível de significância de 5\%.

\section{RESULTADOS}

A partir da anamnese foi detectado que nenhum dos participantes fazia uso de medicamentos ou tinham algum tipo de doença; somente um não fazia uso de nenhum tipo de bebida alcóolica frequentemente e todos praticavam atividade física pelo menos três vezes por semana.
Houve uma diferença estatística significante $(\mathrm{p}=0,0058)$ no tempo de esforço máximo entre os dois testes. A Figura 1 demonstra o tempo máximo de esforço atingido no primeiro teste; com exceção do $4^{\circ}$ participante, que obteve um resultado melhor no segundo teste, os outros nove voluntários tiveram queda no rendimento durante o teste, no qual todos beberam cerveja. A média de todos os participantes foi de $965,8 \pm$ 109,05 segundos no primeiro teste e de 925,9 $\pm 103,85$ segundos no segundo teste.

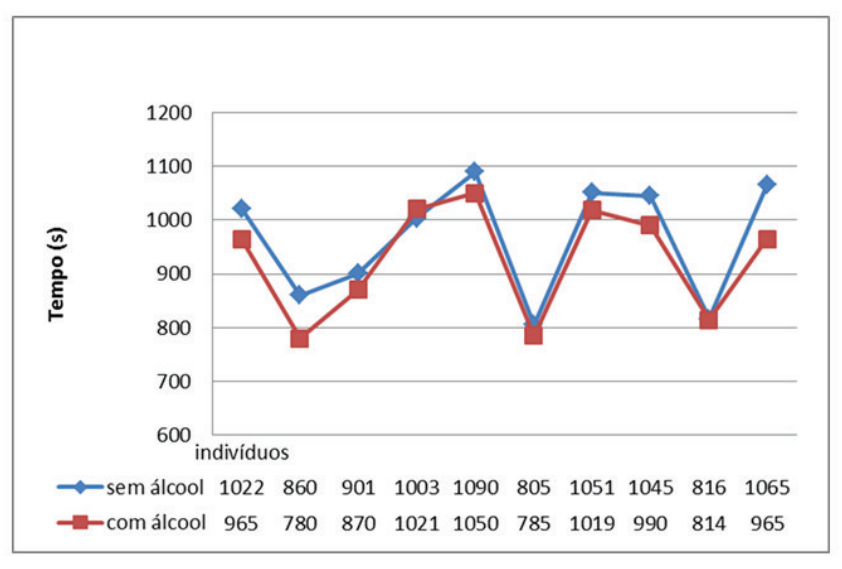

Figura 1. Comparação de tempo de esforço máximo de teste de cada participante nos dois testes

As Figuras 2, 3 e 4 apresentam as médias de frequência cardíaca, aferida a cada minuto durante o teste (dividida em Frequência Cardíaca Inicial, Frequência Cardíaca Aquecimento e Frequência Cardíaca durante o teste). Não houve diferença estatisticamente significativa entre os dois testes $(\mathrm{p}=0,1)$, porém, observando a Figura 1, pode-se concluir que as frequências máximas foram atingidas mais precocemente no teste realizado pelos participantes quando consumiram cerveja.

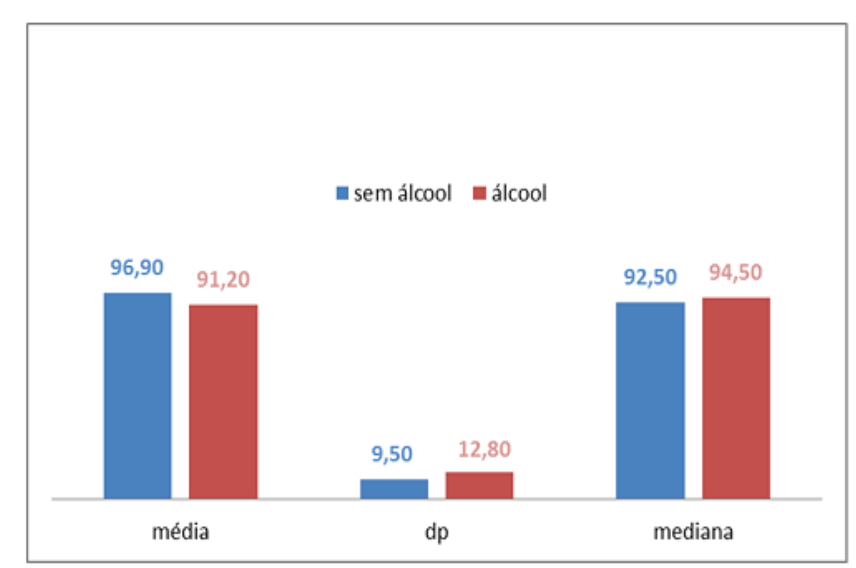

Figura 2. Frequência cardíaca de repouso (média, desvio padrão e mediana). 


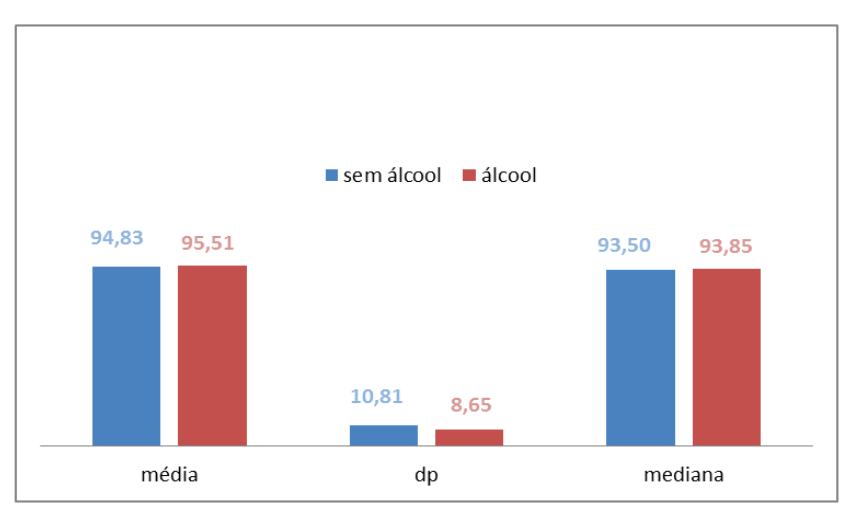

Figura 3. Frequência cardíaca durante 7 minutos de aquecimento (média, desvio padrão e mediana).

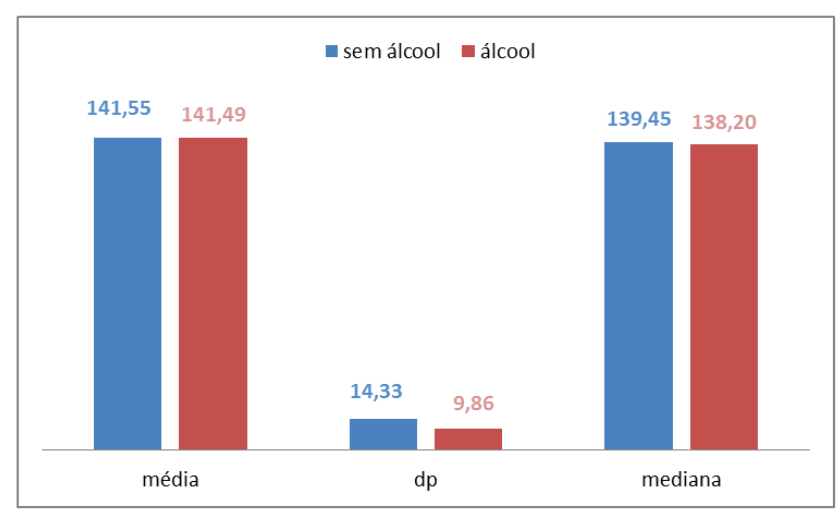

Figura 4. Frequência cardíaca durante o teste de esforço (média, desvio padrão e mediana).

A diferença estatística entre os resultados da Figura 5 foi significante $(p=0,015)$ e demonstra que as potências máximas atingidas durante o teste realizado sem ingestão alcóolica foram maiores em relação ao realizado com ingestão alcóolica pré-teste. O desempenho atingido por seis dos dez participantes foi pior no segundo teste. Somente quatro participantes igualaram os resultados de carga, contudo não conseguiram manter a cadência de pedalada pelo mesmo tempo, como observado na Figura 1.

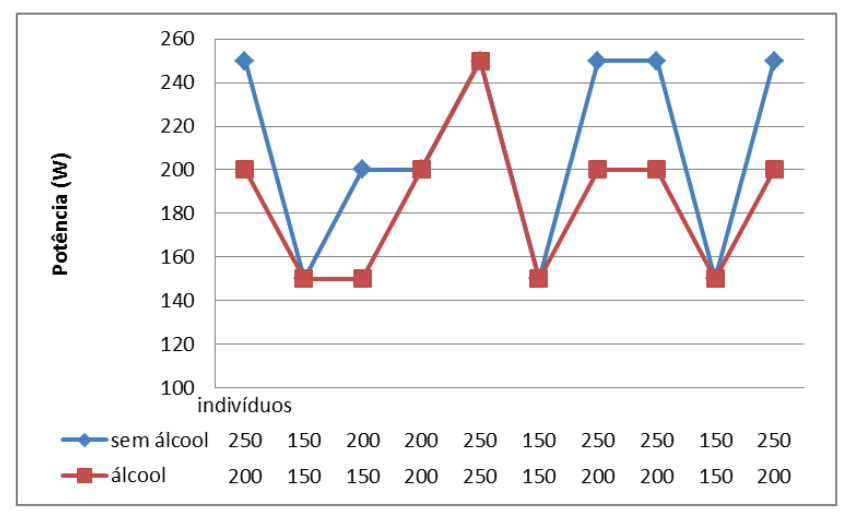

Figura 5. Comparação da potência máxima atingida por cada participante nos dois testes.

\section{DISCUSSÃO}

Estudos semelhantes feitos com atletas, sedentários, pessoas moderadamente treinadas e altamente treinadas mostraram que ao ingerirem álcool antes de um teste de ciclismo tiveram perda no rendimento (SUTTER; SCHUTZ, 2008; LECOULTRE; SCHUTZ, 2009). Ao ingerirem álcool com uma refeição contatou-se o aumento da lipemia, bem como o maior tempo até que voltasse ao normal os índices de gordura no sangue, influenciando os metabolismos de carboidratos e gorduras (SUTTER; SCHUTZ, 2008). Treze ciclistas de resistência treinados, que ingeriram $0,5 \mathrm{ml}$ de etanol por quilo de massa magra durante o segundo teste, tiveram uma decaída nos valores de potência e nos valores de $\mathrm{VO}_{2}$ em relação ao primeiro teste, no qual não utilizaram etanol.

O presente estudo obteve resultados semelhantes. O tempo de teste de esforço máximo e a potência máxima dos voluntários decaíram de um teste para o outro, um dos motivos para a piora de desempenho é que o etanol não poder ser usado como fonte de energia pelo músculo. É no fígado que ocorre a oxidação do álcool, inibindo a gliconeogênese, além de não poder ser estocado como fonte de energia por ser uma substância tóxica, por isso o metabolismo glicolítico, mais utilizado durantes atividades intensas, não é utilizado prejudicando, assim, exercícios que exigem esforço máximo e que foi confirmado neste estudo (OGA, 1996; SUTTER; SCHUTZ, 2008; SHIRREFFS; MAUGHAN, 2006; SUTTER, 2005; MILAM; KETCHAM, 1991; PALACIOS, 2000).

Shirreffs e Maughan (2006), em sua revisão, explicaram que a maior ingestão alcóolica está diretamente ligada à retenção de líquidos; por seu efeito diurético influencia na termoregulação do corpo devido à desidratação provocada pelo álcool, prejudicando a performance em exercícios de endurance, em que tiveram perda de rendimento. Os resultados dos testes obtidos neste estudo também são consistentes a outros testes feitos com corredores treinados de provas rápidas e provas longas (LECOULTRE; SCHUTZ, 2009; MCNAUGHTON; PREECE, 1986; KENDRICK; AFFRIME; LOWENTHAL, 1993). 
Kendrick, Affrime e Lowenthal (1993) observaram em seu estudo, feito com quatro corredores treinados, que a administração de etanol resultou em um significante aumento na resposta da frequência cardíaca nos atletas testados em esteira durante longo período, onde três dos quatro atletas não conseguiram completar o teste após a administração de etanol. Neste estudo, houve uma pequena queda nas médias das frequências cardíacas iniciais e aquelas aferidas a cada minuto durante o aquecimento e durante o teste, contudo não houve diferença estatística significante.

\section{CONSIDERAÇÕES FINAIS}

Conclui-se neste estudo que os efeitos causados pelo consumo de álcool em indivíduos não sedentários contribuíram negativamente no desempenho físico, diminuindo a potência e o tempo no teste de esforço máximo.

\section{REFERÊNCIAS}

CASPERSSEN, C. J.; POWELL, K. E.; CHRSITENSON, G. M. Physical activity exercises and physical fitness: definitions and distinctions for health relates research. Public Health Rep., v. 102, n. 2, p. 126-131, 1985.

JACKSON, A. S.; POLLOCK, M. L. Generalized equations for predicting body density of men. Br J Nutr., v. 40, n. 3 , p. 497-504, 1978.

JACKSON, A. S.; POLLOCK, M. L.; WARD, A. Generalized equations for predicting body density of women. Med Sci Sports Exerc., v. 12, n. 3, p. 175-181, 1980.

KENDRICK, Z. V.; AFFRIME, M. B.; LOWENTHAL, D. T. Effect of etanol on metabolic responses to treadmill runnin in well-trained men. J Clin Pharmacol., v. 33, n. 2, p. 136-139, 1993.

LAMONTE, M. J.; AINSWORTH, B. E. Quantifying energy expenditure and physical activity in the context of dose response. Med Sci Sports Exerc., v. 33, n. 6, p. 370-378, 2001.
LECOULTRE, V.; SCHUTZ, Y. Effect of small dose of alcohol on endurance performance of trained cyclists. Med Sci Sports Exerc., v. 44, n. 3, p. 278-283, 2009.

MARTENS, M. P.; WATSON, I, J. C.; ROYLAND, E. M.; BECK, N. C. Development of the Athlete Drinking Scale. Psychol Addict Behav., v. 19, n. 2, p. 158-164, 2005.

MCNAUGHTON, L.; PREECE, D. Alcohol and its effects on sprint and middle distance running. Br. J. Sports Med., v. 20, n. 3, p. 56-59, 1986.

MILAM, J. R.; KETCHAM, K. Alcoolismo: os mitos e a realidade. São Paulo: NBL, 1991.

NAHAS, M. V. Atividade física, saúde e qualidade de vida: conceitos e sugestões para um estilo de vida ativo. 2. ed. Londrina: Midiograf, 2001.

OGA, S. Fundamentos de toxicologia. São Paulo: Atheneu, 1996.

ORGANIZAÇÂO MUNDIAL DA SAÙDE - OMS. Relatório sobre a saúde no mundo 2001: saúde mental. Genebra: OMS, 2001.

PALACIOS, G. N. Nutrition and physical exercises. Nutr Hosp Madri, v. 15, n. 1, p. 31-40, 2000.

PATE, R. R.; PRATT, M.; BLAIR, S. N. et al. Physical activity and public health: a recomendation from the center for disease control and prevention and the American College os Sports Medicine. JAMA, v. 273, n. 5, p. 402-407, 1995.

RODRIGUEZ, N. R.; DIMARCO, N. M.; LANGLEY, S. Position of the American Dietetic Association, Dietitians os Canada, American College of Sports Medicine: Nutrition and athletic performance. J. Am. Diet. Assoc, v. 109, n. 3, p. 509-527, 2009.

SHIRREFFS, S. M.; MAUGHAN, R. J. The effect of alcohol on athletic performance. Current Sports Medicine Reports, v. 5, n. 1, p. 192-196, 2006.

SUTTER, P. M. Is alcohol consumption a risk factor for weight gain and obesity? Critical Reviews in Clinical Laboratory Sciences, v. 42, n. 3, p. 197-277, 2005. 
SUTTER, P. M.; SCHUTZ, Y. The effect of exercise, alcohol or both combined on health and physical performance. International Journal of Obesity of Lausanne, v. 32, n. 2, p. 48-52, 2008.

WORLD HEALTH ORGANIZATION. WHO. Preventing

Chronic Diseases: a vital investment. Genebra, WHO, 2005.

Recebido em: 19 de fevereiro de 2015 Aceito em: 20 de abril de 2015 